\title{
Intersectional analysis of life stress, incarceration and sexual health risk practices among cisgender Black gay, bisexual and other men who have sex with men in the Deep South of the US: the MARI Study
}

\author{
Dustin T. Duncan ${ }^{\mathrm{A}, \mathrm{G}, \mathrm{H}, *}$, Denton Callander ${ }^{\mathrm{A},{ }^{*}, \text { Lisa Bowleg }}{ }^{\mathrm{B}}$, Su Hyun Park ${ }^{\mathrm{A}}$, \\ Lauren Brinkley-Rubinstein ${ }^{\mathrm{C}}$, Katherine P. Theall ${ }^{\mathrm{D}}$ and DeMarc A. Hickson ${ }^{\mathrm{E}, \mathrm{F}}$ \\ ADepartment of Population Health, NYU School of Medicine, New York, NY 10016, USA. \\ ${ }^{B}$ Department of Psychology, Columbian College of Arts and Sciences, George Washington University, \\ Washington, DC 20052, USA. \\ ${ }^{C}$ Department of Social Medicine, University of North Carolina, Chapel Hill, NC 27516, USA. \\ ${ }^{D}$ Department of Global Community Health and Behavioral Sciences, Tulane University of School of Public \\ Health and Tropical Medicine, New Orleans, LA 70112, USA. \\ ${ }^{E}$ Center for Research, Evaluation and Environmental and Policy Change, My Brother's Keeper Inc., \\ Jackson, MS 39211, USA. \\ FUs Helping Us, People Into Living, Washington, DC 20010, USA. \\ GPresent address: Department of Epidemiology, Columbia University Mailman School of Public Health, \\ New York, NY 10032, USA. \\ ${ }^{\mathrm{H}}$ Corresponding author. Email: dd3018@columbia.edu
}

\begin{abstract}
Objectives: The purpose of the present study was to examine associations between life stress and incarceration history in relation to sexual health risk practices among a sample of cisgender Black gay, bisexual and other men who have sex with men (MSM) in the Deep South. Methods: Using data from a sample of 355 cisgender Black MSM in Mississippi and Georgia, multivariable logistic regression analyses were conducted to examine associations between life stress and sexual risk practices. In addition, we assessed whether stress may interact with experiences of incarceration to influence sexual health risk practices. Results: After controlling for sociodemographic characteristics, stress was associated with some sexual risk practices (e.g. alcohol and drug use during sex and group sex). Further, when an interaction with incarceration was assessed, among participants who had been incarcerated, high compared with low levels of stress were associated with alcohol use during sex (adjusted odds ratio (aOR) 4.59, 95\% confidence interval (CI) 2.11-9.99, $P<0.001$ ), drug use during sex (aOR 3.92, 95\% CI 1.79-8.60, $P<0.001$ ), condomless sex with casual partners (aOR 2.83, 95\% CI 1.31-6.12, $P<0.001$ ), having six or more casual partners (aOR 2.77, 95\% CI 1.09-7.06, $P=0.02$ ) and participating in group sex (aOR 5.67, 95\% CI 2.07-15.51, $P<0.001)$. Stress and incarceration produced a dose-response effect for each association; similar associations with stress were not observed among men who had not been incarcerated. Conclusions: Among people with experiences of incarceration, there are several possible ways our findings could be applied practically, including through safer sex and stress management interventions designed specifically for Black MSM following their release.
\end{abstract}

Additional keywords: health disparities, HIV, intersectionality.

Received 29 March 2019, accepted 5 August 2019, published online 24 January 2020

\section{Introduction}

Stress is a fundamental mechanism underlying a wide variety of health behaviours and outcomes, ${ }^{1}$ including condomless receptive anal intercourse and infection with HIV and other sexually transmissible infections (STIs), ${ }^{2}$ which may undergird health disparities. ${ }^{3}$ Multiple frameworks have been used to explain stress-related health disparities. As articulated by the minority stress model, members of sexual and racial minority populations may experience stress due to stigma associated with their identities and, as a result, have higher stress levels than

\footnotetext{
*These authors contributed equally to the study.
} 
their peers from non-minority groups. ${ }^{4}$ However, limited research has examined relationships between stress and risk for HIV and other STIs among cisgender Black gay, bisexual and other men who have sex with men (MSM), even though in the US this population is at 'high risk' for these infections. ${ }^{5}$ In fact, it has been estimated that nearly $50 \%$ of Black MSM in the US will become infected with HIV over their lifetime, ${ }^{5}$ with HIV particularly prevalent among Black MSM in the Deep South (which includes Louisiana, Mississippi, Alabama, Georgia and South Carolina). ${ }^{6,7}$ Further, rates of chlamydia, gonorrhoea and syphilis among Black MSM in the US are consistently found to outpace their MSM peers of other racial identities, ${ }^{8}$ including in the Deep South. ${ }^{9}$

High rates of HIV and other STIs among Black MSM in the Deep South of the US demand attention to factors and forces that place these men at risk of infection. An experience of incarceration is one factor that has been assessed relative to HIV risk among Black MSM, ${ }^{10}$ described by some as having a 'syndemic connection' with HIV among this population, ${ }^{11}$ although less attention has been paid to incarceration and STIs. ${ }^{12}$ It has been noted that experiences of incarceration are associated with higher-risk sexual practices among Black $\mathrm{MSM}^{13,14}$ and greater instability in the social and sexual networks, ${ }^{15}$ but not necessarily with HIV infection. ${ }^{10}$ This seeming paradox suggests a complexity of the relationship between incarceration and HIV among Black MSM requiring closer attention to factors that mediate their existence. Previous research has highlighted stress as a potential mechanism of health risk following incarceration, ${ }^{16}$ which may be especially relevant for those already seeking to cope with minority stress, but little is known about if or how stress may interact with experiences of incarceration to affect sexual health risk practices.

The purpose of the present study was to examine the relationship between experiences of life stress and sexual health risk behaviours among a sample of Black MSM in the Deep South given that Black MSM in the Deep South experience a range of stressors (racism, homophobia, HIV stigma etc.), using an intersectionality framework that recognises the interconnected nature of social categorisations, such as race, class and gender, as they apply to a given individual or group, regarded as creating overlapping and interdependent systems of disadvantage. Adopting an intersectional approach as a critical analytical framework, we sought to explore whether incarceration among Black MSM was an intersectional position that undermined their capacity to deal with stress and, in turn, was associated with sexual risk practices, including condomless anal sex. In the US, incarceration status is an intersectional position that reflects and affects one's power and privilege, especially for Black men. Stated simply, individual-level experiences of incarceration reflect larger social structures of inequality and discrimination experienced by Black men. Because incarcerated individuals have less power and privilege in American society, we hypothesised that incarcerated Black MSM would be less able to cope with life stress and consequently be more likely to engage in sexual risk behaviours. ${ }^{2}$ For example, incarcerated individuals in the US lose voting rights; in 22 states (including Georgia), felons lose their voting rights during incarceration and for a period of time after, typically while on parole and/or probation.
However, voting rights are automatically restored after this time period. In addition, former felons may have to pay any outstanding fines, fees or restitution before their voting rights are restored. In 12 states (including Mississippi) felons lose their voting rights indefinitely for some crimes or require a governor's pardon in order for voting rights to be restored or face an additional waiting period after completion of sentence (including parole and probation) before voting rights can be restored.

We recognise that Black MSM are typically conceptualised as a homogeneous group, including in intersectionality studies. ${ }^{17}$ Therefore, our approach to intersectionality was twofold. First, by focusing on Black MSM in the Deep South we were able to conduct within-group analyses, as important considerations related to race, gender, sexual orientation and geography were isolated, focusing on a contextualised population as one approach to intersectional analyses. $^{18,19}$ Second, while acknowledging ongoing debates around the best way to incorporate intersectional theory into quantitative work, ${ }^{20}$ we adopted an approach that tests for effect modification via interactions. Although criticised in some quarters as an imperfect representation of intersectionality, ${ }^{21}$ assessing interactions has been supported in others as one way to conduct intersectionality, ${ }^{20}$ which allowed us to approximate it as a framework to guide the use of established and rigorous statistical methods.

\section{Methods \\ Study design}

We undertook a cross-sectional analysis of data collected as part of a study of Black gay, bisexual, and other men who have sex with men (referred to in this paper as 'Black MSM'), called The Ecological Study of Sexual Behaviours and HIV/STIs among African American Men who have sex with Men in in the Southeastern United States, known colloquially as the MARI Study.

\section{Setting}

The MARI Study was established in two cities in the southern part of the US: Jackson, Mississippi and Atlanta, Georgia. Broadly, the study aimed to describe and explore the environmental, behavioural, sociodemographic and HIV risk behaviours among Black gay and bisexual men and included a limited number of Black transgender women. ${ }^{22}$ Participants were given US\$35 (which later increased to US\$50 to bolster participation). Data were collected from July 2013 in Jackson and from December 2014 in Atlanta until the end of 2015.

The MARI Study research protocols were approved by the Sterling Institutional Review Board and all participants provided written informed consent. The secondary analyses reported here were determined to be exempt by the NYU School of Medicine Institutional Review Board.

\section{Participants}

Eligible participants in the MARI Study were cisgender men and transgender women who were aged $\geq 18$ years and who reported sex with at least one man in the 6 months before enrolment. This analysis was restricted to cisgender gay and bisexual men, excluding transgender women given the differences in their likelihood of incarceration ${ }^{14}$ as well as 
documented socioeconomic differences and differences in sexual risk behaviours. ${ }^{23,24}$ Overall, MARI Study recruitment activities involved efforts by local organisations, print advertisements distributed locally to colleges and universities, adult bookstores, bars and clubs, as well as digital advertisements posted on social media and through paid advertisements on geospatial sex and dating applications. Full details on the recruitment methods have been reported previously. ${ }^{22}$

\section{Study survey}

Participants provided information on a range of topics via a computer-assisted survey, including mental health issues, body image, personality traits, religiosity and spirituality, early life environments, social support and neighbourhood environments. Participants also provided sociodemographic information (e.g. age, ethnic identity, sexual orientation, education, employment and income), HIV status, HIV testing and other factors. Incarceration history was assessed with the question 'Have you ever spent any time in a correctional facility, jail, prison, or detention centre?' (yes/no).

\section{Stress}

Participants completed a modified version of the social readjustment rating scale of Holmes and Rahe, ${ }^{25}$ which we used as a proximal marker of life stress. The original version of this well-known scale includes 43 life events rated as requiring a significant change to one's accustomed patterns of living, relatively quantified based on the amount and duration of change required. For example, death of a spouse was ranked as requiring the greatest change (mean adjustment value of 100), whereas other experiences, such as changing schools, were further down the list (mean adjustment value of 20). The scale has been previously challenged for its lack of directionality (many items are presented as neutral, making it impossible to assess whether something added or diminished stress) and a lack of item specification. ${ }^{26}$ It was also not clear whether this scale, developed in the 1960s, would be relevant to our sample, which is demographically and temporally very different to the one in which the scale was validated. With these issues in mind, the study team selected 11 of the items most likely to be relevant to a sample of Black gay and bisexual men and modified them to more specifically address HIV risk behaviours and directionality. For example, instead of the item 'Change in living conditions', participants were asked 'Have you moved to a worse residence or neighbourhood?' Full details of the modified scale are given in Table 1.

Regarding our measure of stress, responses to each of the 11 items were summed using the relative assigned value from Holmes and Rahe's original scale. ${ }^{25}$ To further contextualise stress relative to our sample, the continuous scores were organised into tertiles labelled as 'low stress' (Tertile 1), 'medium stress' (Tertile 2) and 'high stress' (Tertile 3). This provided us with a relative and categorical marker of participant stress in the 12 months before participation.

\section{Sexual risk practices}

Specific to the 12 months before participation, participants completed items on drug or alcohol use in the context of
Table 1. Experiences of Black gay, bisexual and other men who have sex with men of stressful life events ${ }^{A}$ in the 12 months before participation $(n=355)$

Data are given as $n(\%)$. Stress was divided into three tertiles, based on stress scores: low (Tertile 1, scores 0-62; mean \pm s.d. score $12.20 \pm 19.27$ ); medium (Tertile 2; scores 67-139; mean \pm s.d. score $98.51 \pm 26.43$; and high (Tertile 3; scores $141-469$; mean \pm s.d. score $233.85 \pm 76.53$ )

\begin{tabular}{lc}
\hline Total score & \\
$\quad$ Low stress & $118(33.2)$ \\
$\quad$ Medium stress & $118(33.2)$ \\
High stress & $119(33.5)$ \\
Scale item (relative score) & $47(13.2)$ \\
$\quad$ Had a divorce/separation from your mate/spouse (69) & $202(56.9)$ \\
$\quad$ Anyone close to you died (67) & $47(13.2)$ \\
$\quad$ Have you had serious injury/illness that started or got & \\
$\quad$ worse in last year (53) & $49(13.8)$ \\
$\quad$ Been a victim of a serious physical attack/mugging any & \\
$\quad$ type of assault (53) & $81(22.8)$ \\
Found out that you are HIV positive/lost a loved one due & \\
$\quad$ to HIV/AIDS (49) & $21(5.9)$ \\
$\quad$ Retired from a job when you did not want to (45) & $132(37.2)$ \\
Family member/close friend had major illness/injury (44) & $76(21.4)$ \\
$\quad$ Been robbed or was home burglarised (25) & $54(15.2)$ \\
$\quad$ Moved to a worse residence/neighbourhood (23) & $110(31.0)$ \\
$\quad$ You/anyone in your household lost a job (23) & $61(17.2)$ \\
$\quad$ House been shot at or gunfire in neighbourhood (18) & \\
\hline
\end{tabular}

${ }^{\text {A }}$ Scale items modified from the social readjustment rating scale of Holmes and Rahe. ${ }^{25}$

sex, condomless anal sex with casual partners and the number of sexual partners. Participants were also asked whether they had recently participated in group sex and, at their most recent sexual encounter, if they had asked their partner about their HIV status.

Risk practices associated with HIV were dichotomously categorised (yes/no) based on each participant's self-reported behaviour during the 12 months before study participation. This included any alcohol use in the context of sex, drug use in the context of sex, condomless sex with casual partners (consistent or inconsistent condom use), numbers of sexual partners $(<6$ or $\geq 6$ male partners), group sex participation (yes/no) and whether they had asked their most recent casual sex partner his HIV status (yes/no). These six factors were used as the outcome measure in the multivariate analyses given their associations with HIV infection risk among Black MSM. ${ }^{27}$

\section{Statistical analyses}

Our analysis was restricted to cisgender male participants who completed all 11 of the modified social readjustment items and provided information on incarceration. We conducted multivariable logistic regression analyses with each of the risk practices described above, controlling for age, sexual orientation, level of education, employment status, income, HIV status and city of recruitment. The categorical indicator of stress was included in all models. To directly address our research question whether stress may interact with experiences of incarceration to influence sexual health risk practices, these analyses were also conducted with a test for interactions between those two variables as well as 
examination of stratum-specific estimates (i.e. associations by no history of incarceration vs history of incarceration).

Statistical significance was set at two-tailed $P<0.05$ and all analyses were performed using Stata Version 15.0 (StataCorp LLC, College Station, TX, USA).

\section{Results}

Of the 386 individuals who participated in the MARI Study, the present analysis was performed on the 355 (92.0\%) cisgender men who completed all 11 stress items and provided details on incarceration. The sociodemographic characteristics of the sample are given in Table 2. Participants ranged in age from 16 to 62 years, with a mean ( \pm s.d.) age of $30.37 \pm 11.35$ years. Only $2.3 \%$ identified as Latino. Most participants were unemployed (54.9\%) and $129(36.3 \%)$ reported previous incarceration. Men also commonly reported the use of alcohol and other drugs in the context of sex $(49.3 \%$ and $36.9 \%$ respectively), whereas over one-third reported condomless anal sex with casual partners $(36.6 \%)$.

Regarding stress, the range of participant scores was 0-469 of a possible maximum score of 475 , with a mean ( \pm s.d.) score of $113.59 \pm 102.66$. Acceptable internal consistency was observed among these modified items $(\alpha=0.71)$. In all, 85 participants $(22.6 \%)$ reported experiencing none of the stressful life events included in our survey, whereas a small number $(n=4 ; 1.1 \%)$ had experienced almost all 11 items. As outlined in Table 1, the most commonly reported stressful event for participants was the death of someone to whom they were close $(56.9 \%)$, followed by having a family member or friend experience a major illness $(37.2 \%)$. These were also ranked among the most stressful life events in the original scale. ${ }^{25}$

The findings of the multivariate analyses are detailed in Table 3. Compared with men who reported low stress in the previous year, those with high levels of stress were more likely to report alcohol and drug use in the context of sex as well as participation in group sex. Interestingly, similar associations were not observed among men with medium levels of life stress. In the subsequent interactional analysis, incarceration interacted with medium and high levels of stress to produce associations with alcohol and drug use, which appeared to be a dose-response relationship. Having a high level of stress in conjunction with previous incarceration was associated with all the sexual risk practices assessed in this study, except for not asking one's partner about his HIV status. In particular, compared with low levels of stress, high levels were associated with alcohol use during sex (adjusted odds ratio

Table 2. Demographics, social characteristics and sexual risk behaviours among cisgender Black gay, bisexual and other men who have sex with men in the Deep South $(n=355)$ Unless indicated otherwise, data are given as $n(\%)$

\begin{tabular}{lc}
\hline Mean ( \pm s.d.) age (years) & $30.37 \pm 11.35$ \\
Latino or Hispanic-identified & $8(2.3)$ \\
Sexual orientation & \\
$\quad$ Gay/homosexual & $241(67.9)$ \\
Bisexual & $95(26.8)$ \\
Other & $19(5.4)$ \\
Highest level of education & \\
$\quad$ Some high school or completed high school & $146(41.1)$ \\
$\quad$ Some college & $139(39.2)$ \\
Bachelor's degree and other higher degree & $70(19.7)$ \\
Currently unemployed & $195(54.9)$ \\
Any previous incarceration & $129(36.3)$ \\
Annual household income (US\$) & \\
$\quad$ 5000 & $135(38.0)$ \\
$5000-15999$ & $103(29.0)$ \\
$\geq 16000$ & $110(31.0)$ \\
No response & $7(2.0)$ \\
City of recruitment & \\
Jackson & $213(60.0)$ \\
Atlanta & $142(40.0)$ \\
HIV positive & $132(36.2)$ \\
In the 12 months before participation: & \\
Alcohol use in the context of sex & $175(49.3)$ \\
Drug use in the context of sex & $131(36.9)$ \\
Condomless anal sex with casual partners & A \\
$\geq 6$ casual male partners & $130(36.6)$ \\
Participated in group sex & $67(19.5)$ \\
Asked last casual partner's HIV status & $58(16.3)$ \\
\hline A Defined 'nev' or 'sometimes' using a con & $181(51.0)$ \\
\hline
\end{tabular}

A Defined as 'never' or 'sometimes' using a condom with casual sex partners.

Table 3. Association between stressful life events in the 12 months before study participation and sexual risk behaviours among cisgender Black gay, bisexual and other men who have sex with men in the Deep South according to previous incarceration status $(n=355)$

Data are given as adjusted odds ratios with $95 \%$ confidence intervals in parentheses. ${ }^{*} P<0.05$; $* * P<0.001$. Stress was divided into three tertiles, based on stress scores: low (Tertile 1, scores 0-62; mean \pm s.d. score $12.20 \pm 19.27$ ); medium (Tertile 2; scores 67-139; mean \pm s.d. score $98.51 \pm 26.43$; and high (Tertile 3; scores 141-469; mean \pm s.d. score $233.85 \pm 76.53$ ). Note, all regression analyses controlled for age, education, annual income, HIV status, recruitment city, employment status, ethnicity sexual orientation, and incarceration status (except models stratified by incarceration status)

\begin{tabular}{|c|c|c|c|c|c|c|}
\hline \multirow[t]{3}{*}{ In the previous 12 months: } & \multicolumn{2}{|c|}{ All $(n=355)$} & \multicolumn{4}{|c|}{ Previous incarceration } \\
\hline & \multirow[b]{2}{*}{ Medium stress ${ }^{\mathrm{A}}$} & \multirow[b]{2}{*}{ High stress ${ }^{\mathrm{A}}$} & \multicolumn{2}{|c|}{ Yes $(n=129)$} & \multicolumn{2}{|c|}{ No $(n=226)$} \\
\hline & & & Medium stress ${ }^{\mathrm{A}}$ & High stress ${ }^{A}$ & Medium stress ${ }^{\mathrm{A}}$ & High stress ${ }^{\mathrm{A}}$ \\
\hline Alcohol in the context of sex & $1.61(0.93-2.79)$ & $2.24(1.25-4.04)^{*}$ & $3.87(1.64-9.07)^{* *}$ & $4.59(2.11-9.99)^{* *}$ & $1.45(0.76-2.75)$ & $2.18(1.04-4.58)^{*}$ \\
\hline Drugs in the context of sex & $0.97(0.53-1.74)$ & $1.96(1.07-3.59)^{*}$ & $3.39(1.44-8.00)^{* *}$ & $3.92(1.79-8.60)^{* *}$ & $0.80(0.38-1.69)$ & $2.63(1.20-5.76)^{*}$ \\
\hline Condomless anal $\mathrm{sex}^{\mathrm{B}}$ & $1.15(0.65-2.03)$ & $1.56(0.86-2.84)$ & $1.38(0.57-3.30)$ & $2.83(1.31-6.12)^{* *}$ & $1.49(0.75-2.97)$ & $1.54(0.70-3.38)$ \\
\hline$\geq 6$ casual sex partners & $0.89(0.43-1.85)$ & $1.24(0.60-2.59)$ & $1.96(0.69-5.60)$ & $2.77(1.09-7.06)^{*}$ & $0.92(0.37-2.27)$ & $1.28(0.48-3.38)$ \\
\hline Participated in group sex & $1.25(0.52-2.98)$ & $4.59(2.06-10.23)^{* *}$ & $1.21(0.32-4.55)$ & $5.67(2.07-15.51)^{* *}$ & $1.26(0.45-3.54)$ & $3.85(1.40-10.59)^{* *}$ \\
\hline Asked partner's HIV status ${ }^{\mathrm{C}}$ & $1.16(0.62-2.18)$ & $1.0(0.52-1.93)$ & $1.16(0.46-2.97)$ & $0.82(0.35-1.91)$ & $1.24(0.58-2.61)$ & $1.30(0.54-3.12)$ \\
\hline
\end{tabular}

${ }^{\mathrm{A}}$ Reference category $=$ low stress $($ Tertile 1$)$.

${ }^{\mathrm{B}}$ Any condomless sex with a casual partner.

${ }^{\mathrm{C}}$ Most recent anal sex encounter only. 
(aOR) 4.59, 95\% confidence interval (CI) 2.11-9.99, $P<0.001$ ), drug use during sex (aOR 3.92, 95\% CI 1.79-8.60, $P<0.001)$, condomless sex with casual partners (aOR 2.83, 95\% CI 1.31-6.12, $P<0.001$ ), having six or more casual partners (aOR 2.77, 95\% CI 1.09-7.06, $P=0.02$ ) and participating in group sex (aOR 5.67, 95\% CI 2.07-15.51, $P<0.001)$. Stress and incarceration produced a doseresponse effect for each association. Although previous incarceration interacting with a high degree of stress was associated with condomless anal sex and having a high number of partners, similar associations were not observed among previously incarcerated men who experienced lower levels of stress, or among those with no experience of incarceration, regardless of stress level.

\section{Discussion}

Intersectionality matters when assessing HIV risk among MSM in the US Deep South. Among Black MSM in the US Deep South, stress was associated with several sexual health risk practices, notably alcohol and drug use in the context of sex and participating in group sex. In addition, being previously incarcerated interacted with Black men's experiences of stress. A history of incarceration strengthened the extant associations and was associated with having more sex partners and condomless anal sex with casual partners. Stress interacting with experiences of incarceration appears to be associated with sexual health practices among Black MSM in the Deep South.

The results of the present study are consistent with earlier research, including the association between stress and various risks to sexual health among Black MSM. ${ }^{28,29}$ Perhaps more interestingly is our finding that a history of incarceration seemed to significantly exacerbate the effects of stress, in several cases linking stress to risk in a way that would have otherwise remained undiscovered. One interpretation of these findings is that Black MSM who have been in jail or prison may struggle to deal with their experiences of stress, resulting in practices that may offset the stress but introduce risk for HIV and other STIs. This finding could also mean that the experience of incarceration exacerbates stress, and the mechanism for this may be the negative effect that incarceration has on other known social determinants of health (housing, social support and relationships, employment) via a restriction of rights following a return to the community.

There is a wealth of literature that has documented the poor health outcomes that follow after release from incarceration, including poor care for chronic conditions, ${ }^{30}$ death $^{31}$ and, specific to HIV and STIs, high rates of condomless sex and substance use. ${ }^{32-34}$ For example, among a sample of HIVpositive MSM, in analyses adjusting for sociodemographic factors, past-year and prior incarceration were strongly associated with past 12-month injection drug use, multiple sex partnerships and condomless sex in the context of substance use. ${ }^{34}$ In addition, these analyses found that pastyear incarceration was strongly associated with alcohol and non-injecting drug use. ${ }^{34}$

By illuminating the interactions between incarceration and stress as a mechanism for sexual risk practices, this study builds on this earlier work and helps enhance our understandings of why similar experiences of stress may prompt risk practices whereas others do not. Incarceration and its subsequent disruption of access and restriction of rights could explain why incarceration compounds stress. Indeed, beyond Black men being most likely to be arrested and harassed by police, intersectional oppression does not end when they are released from prison. As an example, Black people in particular are much less likely than their White peers to be hired for a job after they are released and, when employed after incarceration, earn less money than their White peers. ${ }^{35-37}$ Among people with experiences of incarceration, there are several possible ways this information could be applied practically. For one, given that incarceration is symptomatic of larger social structure inequality, it seems prudent to intervene there. That is, there is a dire need for policy and legislative change to address why Black men are more likely than other groups of men to be incarcerated in the first place. ${ }^{37}$ This not only applies to Black men, but also Black MSM, because research shows that Black MSM are more likely be incarcerated than MSM of other racial backgrounds. ${ }^{38}$ In addition, it seems that an intervention aimed at improving stress management (e.g. reducing stress levels) among Black gay and bisexual men who have been incarcerated may reduce engagement in sexual risk practices, especially for those in the Deep South. Incarceration rates are highest in the South, making these findings especially important in that region. Knowing that this association exists (in this population), public health officials and HIV/AIDS service providers could consider screening for recent incarceration and providing access to more supportive services (e.g. mental health services) that can alleviate stress and promote HIV prevention among Black MSM.

This study highlighted experiences that are associated with sexual risk among Black MSM in the Deep South. By using an intersectional framework, ${ }^{18,19}$ we were able to characterise experiences of sexual risk taking that would have otherwise remained unexamined because we would not have thought to examine incarceration status as an intersectional position. Although this study does provide meaningful findings to the literature on stress, incarceration and sexual risk behaviours, it is also limited in a few key ways. First, although our measure of stress used a modified version of a previously validated scale, it is a measure of general stress that may be less salient to the lives of Black men. Future research should seek to develop, validate and implement scales of stress applicable to Black MSM, including the examination of minority-, oppression- and intersectional-related stress. Second, the convenience sample upon which this study draws is limited to only two cities in the Deep South, a large and diverse part of the US, and it is unclear to what degree (if any) these findings can be applied elsewhere. Third, as a cross-sectional analysis, we are unable to comment on the directionality of the relationships observed here, also because of how the study variables were measured. It is important to note that incarceration was assessed as 'ever' and the risk behaviours and stress was assessed as 'past year'. Therefore, it is possible that some of the past-year HIV risk behaviours occurred during past-year incarceration. In addition, a history of incarceration may be a crude measure. There are many nuances to incarceration that this study missed, such as past-year incarceration versus any incarceration and variations in incarceration conditions, which should be examined in 
future research in addition to other intersectional positions (e.g. nativity status $)^{39}$ related to power and inequality that may be salient to the association between stress and sexual risk behaviours. Further research on the sexual health risks and stress management skills of Black gay and bisexual men before and after incarceration is warranted. Importantly, future research also needs to examine policy and legislative changes to address why Black men are more likely than other groups of men to be incarcerated, including as related to variations in health, such as sexual health risks.

\section{Conflicts of interest}

The authors declare no conflicts of interest.

\section{Acknowledgements}

At the time of analysis and writing, Dustin Duncan was supported, in part, by grants from the National Institute on Minority Health and Health Disparities (Grant no. R01MD013554), National Institute on Mental Health (Grant no. R01MH112406), National Institute on Drug Abuse (Grant no. R03DA039748) and the Centers for Disease Control and Prevention (CDC; Grant no. U01PS005122). This project was supported by a grant from the CDC (Grant no. U01PS003315 to DeMarc A. Hickson). In addition, DeMarc A. Hickson was supported, in part, by a grant from the National Institute on Mental Health (Grant no. R25MH083620). The authors thank the participants for engaging in this study.

\section{References}

1 Schneiderman N, Ironson G, Siegel SD. Stress and health: psychological, behavioral, and biological determinants. Annu Rev Clin Psychol 2005; 1: 607-28. doi:10.1146/annurev.clinpsy.1.102803. 144141

2 Hulland EN, Brown JL, Swartzendruber AL, Sales JM, Rose ES, DiClemente RJ. The association between stress, coping, and sexual risk behaviors over 24 months among African-American female adolescents. Psychol Health Med 2015; 20: 443-56. doi:10.1080/ 13548506.2014.951369

3 Sternthal MJ, Slopen N, Williams DR. Racial disparities in health: how much does stress really matter? Du Bois Rev 2011; 8: 95-113. doi:10.1017/S1742058X11000087

4 Meyer IH. Prejudice, social stress, and mental health in lesbian, gay, and bisexual populations: conceptual issues and research evidence. Psychol Bull 2003; 129: 674-97. doi:10.1037/0033-2909.129.5.674

5 Centers for Disease Control and Prevention. Lifetime risk of HIV diagnosis - press release: Half of black gay men and a quarter of Latino gay men projected to be diagnosed within their lifetime. 2016. Available online at: https://www.cdc.gov/nchhstp/newsroom/2016/ croi-press-release-risk.html [verified 1 May 2019].

6 Lieb S, Prejean J, Thompson DR, Fallon SJ, Cooper H, Gates GJ, et al. HIV prevalence rates among men who have sex with men in the Southern United States: population-based estimates by race/ethnicity. AIDS Behav 2011; 15: 596-606. doi:10.1007/s10461-010-9820-y

7 Reif SS, Whetten K, Wilson ER, McAllaster C, Pence BW, Legrand $\mathrm{S}$, et al. HIV/AIDS in the Southern USA: a disproportionate epidemic. AIDS Care 2014; 26: 351-9. doi:10.1080/09540121. 2013.824535

8 Sullivan PS, Peterson J, Rosenberg ES, Kelley CF, Cooper H, Vaughan A, et al. Understanding racial HIV/STI disparities in black and white men who have sex with men: a multilevel approach. PLoS One 2014; 9: e90514. doi:10.1371/journal.pone. 0090514
9 Aral SO, O'Leary A, Baker C. Sexually transmitted infections and HIV in the Southern United States: an overview. Sex Transm Dis 2006; 33: S1-5. doi:10.1097/01.olq.0000223249.04456.76

10 Brewer RA, Magnus M, Kuo I, Wang L, Liu TY, Mayer KH. Exploring the relationship between incarceration and HIV among black men who have sex with men in the United States. $J$ Acquir Immune Defic Syndr 2014; 65: 218-25. doi:10.1097/01.qai. $0000434953.65620 .3 \mathrm{~d}$

11 McCarthy E, Myers JJ, Reeves K, Zack B. Understanding the syndemic connections between HIV and incarceration among African American men, especially African American men who have sex with men. In: Wright ER, Carnes $\mathrm{N}$, editors. Understanding the HIV/AIDS epidemic in the United States: the role of syndemics in the production of health disparities. Cham: Springer International Publishing; 2016. pp. 217-40.

12 Watson DL, Liao C, Ridgway J, Schneider JA. Behavioral characteristics and their association with syphilis seroprevalence among a cohort of young Black men who have sex with men. Int J STD AIDS 2019; 30: 396-403. doi:10.1177/0956462418816449

13 Bland SE, Mimiaga MJ, Reisner SL, White JM, Driscoll MA, Isenberg D, et al. Sentencing risk: history of incarceration and HIV/STD transmission risk behaviours among Black men who have sex with men in Massachusetts. Cult Health Sex 2012; 14 : 329-45. doi:10.1080/13691058.2011.639902

14 Brewer RA, Magnus M, Kuo I, Wang L, Liu TY, Mayer KH. The high prevalence of incarceration history among Black men who have sex with men in the United States: associations and implications. Am J Public Health 2014; 104: 448-54. doi:10.2105/AJPH.2013.301786

15 Schneider JA, Lancki N, Schumm P. At the intersection of criminal justice involvement and sexual orientation: dynamic networks and health among a population-based sample of young Black men who have sex with men. Soc Networks 2017; 51: 73-87. doi:10.1016/j. socnet.2017.04.001

16 Brinkley-Rubinstein L. Incarceration as a catalyst for worsening health. Health \& Justice 2013; 1: 3. doi:10.1186/2194-7899-1-3

17 Bowleg L. 'Once you've blended the cake, you can't take the parts back to the main ingredients': Black gay and bisexual men's descriptions and experiences of intersectionality. Sex Roles 2013; 68: 754-67. doi:10.1007/s11199-012-0152-4

18 Bowleg L. The problem with the phrase women and minorities: intersectionality - an important theoretical framework for public health. Am J Public Health 2012; 102: 1267-73. doi:10.2105/ AJPH.2012.300750

19 Bowleg L, Del Rio-Gonzalez AM, Holt SL, Perez C, Massie JS, Mandell JE, et al. Intersectional epistemologies of ignorance: how behavioral and social science research shapes what we know, think we know, and don't know about U.S. Black men's sexualities. $J$ Sex Res 2017; 54: 577-603. doi:10.1080/00224499.2017.1295300

20 Bauer GR. Incorporating intersectionality theory into population health research methodology: challenges and the potential to advance health equity. Social Science \& Medicine 2014; 110: 10-17. doi:10.1016/j.socscimed.2014.03.022

21 Bowleg L. When Black + lesbian + woman $\neq$ Black lesbian woman: the methodological challenges of qualitative and quantitative intersectionality research. Sex Roles 2008; 59: 312-25. doi:10.1007/ s11199-008-9400-z

22 Hickson DA, Truong NL, Smith-Bankhead N, Sturdevant N, Duncan DT, Schnorr J, et al. Rationale, design and methods of the ecological study of sexual behaviors and HIV/STI among African American men who have sex with men in the Southeastern United States (the MARI Study). PLoS One 2015; 10: e0143823. doi:10.1371/journal. pone. 0143823

23 Ezell JM, Ferreira MJ, Duncan DT, Schneider JA. The social and sexual networks of Black transgender women and Black men who 
have sex with men: results from a representative sample. Transgend Health 2018; 3: 201-9. doi:10.1089/trgh.2018.0039

24 Siembida EJ, Eaton LA, Maksut JL, Driffin DD, Baldwin R. A comparison of HIV-related risk factors between Black transgender women and Black men who have sex with men. Transgend Health 2016; 1: 172-80. doi:10.1089/trgh.2016.0003

25 Holmes TH, Rahe RH. The social readjustment rating scale. Journal of Psychosomatic Research 1967; 11: 213-18. doi:10.1016/ 0022-3999(67)90010-4

26 Mechanic D. Some problems in the measurement of stress and social readjustment. J Human Stress 1975; 1: 43-8. doi:10.1080/0097840X. 1975.9939546

27 McNair OS, Gipson JA, Denson D, Thompson DV, Sutton MY, Hickson DA. The associations of resilience and HIV risk behaviors among Black gay, bisexual, other men who have sex with men (MSM) in the Deep South: the MARI Study. AIDS Behav 2018; 22: 1679-87. doi:10.1007/s10461-017-1881-8

28 Voisin DR, Hotton AL, Schneider JA. The relationship between life stressors and drug and sexual behaviors among a population-based sample of young Black men who have sex with men in Chicago. AIDS Care 2017; 29: 545-51. doi:10.1080/09540121.2016.1224303

29 Quinn K, Voisin DR, Bouris A, Schneider J. Psychological distress, drug use, sexual risks and medication adherence among young HIVpositive Black men who have sex with men: exposure to community violence matters. AIDS Care 2016; 28: 866-72. doi:10.1080/ 09540121.2016.1153596

30 Fox AD, Anderson MR, Bartlett G, Valverde J, Starrels JL, Cunningham $\mathrm{CO}$. Health outcomes and retention in care following release from prison for patients of an urban post-incarceration transitions clinic. J Health Care Poor Underserved 2014; 25: 1139-52. doi:10.1353/hpu.2014.0139

31 Binswanger IA, Stern MF, Deyo RA, Heagerty PJ, Cheadle A, Elmore JG, et al. Release from prison - a high risk of death for former inmates. $N$ Engl $J$ Med 2007; 356: 157-65. doi:10.1056/ NEJMsa064115

32 Adams LM, Kendall S, Smith A, Quigley E, Stuewig JB, Tangney JP. HIV risk behaviors of male and female jail inmates prior to incarceration and one year post-release. AIDS Behav 2013; 17: 2685-94. doi:10.1007/s10461-011-9990-2

33 Brinkley-Rubinstein L, Parker S, Gjelsvik A, Mena L, Chan PA, Harvey $\mathrm{J}$, et al. Condom use and incarceration among STI clinic attendees in the Deep South. BMC Public Health 2016; 16: 971. doi:10.1186/s12889-016-3590-z

34 Khan MR, McGinnis KA, Grov C, Scheidell JD, Hawks L, Edelman EJ, et al. Past year and prior incarceration and HIV transmission risk among HIV-positive men who have sex with men in the US. AIDS Care 2019; 31: 349-56. doi:10.1080/09540121.2018.1499861

35 Western B, Sirois C. Racial inequality in employment and earnings after incarceration. 2017. Available online at: https://scholar.harvard. edu/files/brucewestern/files/racial_inequality_in_employment_ and_earnings_after_incarceration.pdf [verified 2 December 2019].

36 Abram KM, Azores-Gococo NM, Emanuel KM, Aaby DA, Welty LJ, Hershfield JA, et al. Sex and racial/ethnic differences in positive outcomes in delinquent youth after detention: a 12-year longitudinal study. JAMA Pediatr 2017; 171: 123-32. doi:10.1001/ jamapediatrics.2016.3260

37 Western B. The impact of incarceration on wage mobility and inequality. Am Sociol Rev 2002; 67: 526-46. doi:10.2307/3088944

38 Anderson-Carpenter KD, Fletcher JB, Reback CJ. Associations between methamphetamine use, housing status, and incarceration rates among men who have sex with men and transgender women. J Drug Issues 2017; 47: 383-95. doi:10.1177/0022042617696917

39 Viruell-Fuentes EA, Miranda PY, Abdulrahim S. More than culture: structural racism, intersectionality theory, and immigrant health. Social Science \& Medicine 2012; 75: 2099-106. doi:10.1016/ j.socscimed.2011.12.037 